\title{
FluidDynamics Library for Coarse-Grid CFD-Simulation in Modelica
}

\author{
Dr. Stefan Wischhusen ${ }^{1}$ Timo Tumforde ${ }^{1} \quad$ Hans-Herrmann Wurr $^{1}$ \\ ${ }^{1}$ XRG Simulation GmbH, Germany, \{wischhusen, tumforde@xrg-simulation.de\}
}

\begin{abstract}
This paper describes the content and the use of the new FluidDynamics Library which can be applied to carry out CFD simulations using Modelica as an open modelling language. Typical applications until now have been in automotive, aircraft and buildings development. In this paper a fire dynamics and smoke removal simulation is presented. These simulations are very important in the process of approving a building permission. The FluidDynamics Library helps to identify promising ventilation and control setups and speeds up the simulation process significantly.

Keywords: CFD, Coarse grid, Computational-FluidDynamics, Navier-Stokes, FluidDynamics Library
\end{abstract}

\section{Introduction}

For simulations of air-conditioned spaces different approaches can be suitable. In 1D-simulation-tools often a single node model is used to model the mass and energy balance for a complete room or building. Those "Lumped"-models are designed for quick simulations of longer time intervals and are supplied by many free or commercial Modelica libraries on the market (examples: HumanComfort Library, AIX Library, Buildings Library). For a more detailed analysis of the inhomogeneous air states and air velocities within the compartment the user has to model the air flow between the discrete volumes, or else he has to establish Navier-Stokes equations which can describe the air flow in 3D. Those Navier-Stokes-based equations are implemented in free or commercial CFD software, like OpenFoam, Ansys Fluent, Star CCM+ and so on. For a combined calculation of both modelling approaches, an interfacing software (Middleware) is required, which handles the exchange of variables at the model boundaries or connections (i.e., inlets and outlets of the air spaces). For this purpose TISC [TIS(2018)] or MpCCI [MPC(2018)] may be used. Although the coupling works fine in general, it requires up to three software licenses. Even in case of a built-in solution (e.g. Ansys Simplorer) a coupled solution requires more computational resources and/or the decoupled solution and data exchange at discrete time points may generate balance failures.

A Modelica-based coarse grid CFD-solution has the following advantages:

1. Save time by faster Modelica simulations

2. Instant simulation success through convergence control of the variable-step solver

3. Reduced license costs with only one simulation software when coupled to Modelica models

4. Reduced elapse time for iterative work and control design loops through faster simulations

5. Model customization since the code is open

6. Efficient modeling through symbolic manipulation of the Modelica source code

\section{FluidDynamics Library Overview}

The FluidDynamics Library, available in Dymola 2018 FD01, consists of the following packages:

- Basics

- Weather

- Zones

- $\mathrm{CFD}$

- Examples

The Basics package supplies all fundamental definitions which are shared by several models, such as records, icons or functions.

The integrated weather model of the Weather Package is able to read arbitrary weather data tables and provides the interpolation to the zone model. Moreover, the model can convert the intensity of radiation to the effective area according to its spatial orientation. All 


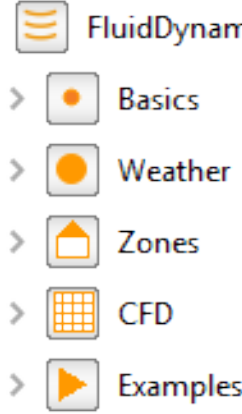

Figure 1. Package overview of the FluidDynamics Library

necessary weather information is bundled and sent to the zone model via a standardized weather connector.

The Zones Package contains models to develop mobile or stationary applications. Mobile applications can be built with models e.g. for aircraft [Michaelsen(2015)] vehicle cabins. All models can individually be built and fitted. The Modelica Code is readable.

The CFD package contains a Modelica-based, three-dimensional grid model. The model is composed of cubic cells. Each cell, which is used to apply the finite-volume-method, may represent a solid or an air cell. The energy and mass balances are calculated in energy cells, while the mass and heat flows are calculated in the so-called flow cell. Here, the influence of the turbulence, the shear forces acting to the air, the gravity and the buoyancy force is taken into account. By using the Navier-Stokes equations, as found in CFD simulation software, realistic flow conditions can be calculated. At the edges of the grid standard interfaces allow an easy connection with models from other libraries. Thus, e.g. a whole building can be represented by one-dimensional wall models from the Zones package connected to the interior represented by the CFD grid model. On demand one can easily exchange the grid model with a lumped volume model for the air side. Of course, Modelica.Fluid interfaces of the Modelica Standard Library are also available. Moreover, symmetric and periodic boundary conditions can be defined in order to reduce the computational effort for larger rooms. Furthermore, the grid model contains all geometric information about the radiation exchange between surfaces. Thus, in advance the view factors to determine the thermal radiation between the surface pairs are calculated by the software, which is required to determine for example internal shading. The spacing and relative orientation of the surfaces are relevant for the exchanged heat radiation.

The Examples package contains models for demonstration of the library capabilities and typical applications.

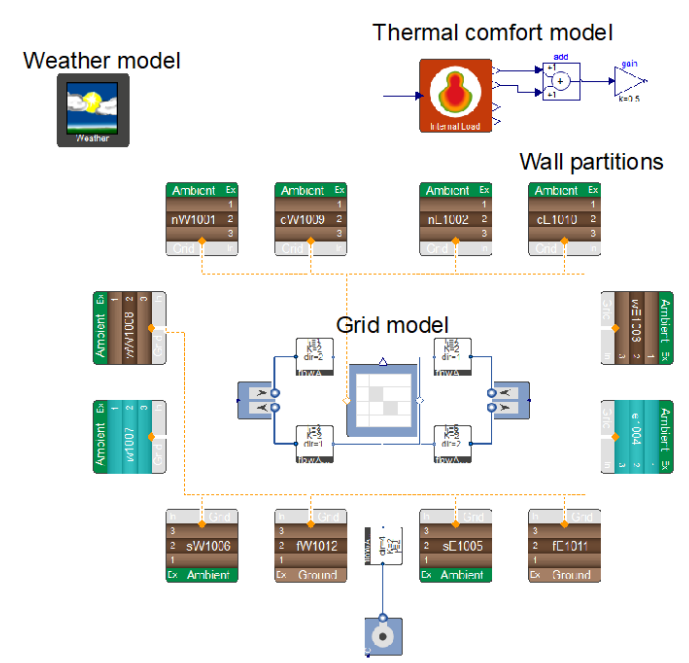

Figure 2. Building example model from Examples package

\subsection{Coarse-grid CFD model}

When modelling a viscous, heat conducting flow, the Navier-Stokes equations are the basic governing equations. They consist of the continuity equation which represents mass conservation, momentum equation which is derived from Newton's Law of Motion and the energy equation which stands for energy conservation. Since the analytical solution is obtainable in only a limited number of cases, one is forced to approximate these equations to obtain workable results. Therefore, using one of the discretisation methods, Navier-Stokes equations in their differential or integro-differential form are transformed into a set of algebraic equations. Discretised equations are numerically solved in a number of discrete points in space and time. There are multiple numerical approaches to CFD modelling including Finite Volume Method, Finite Element Method and Finite Difference Method. Out of them all, the Finite Volume Method is most widely used for fluid flow problems since it is conservative by default as long as the surface integrals are equal for all control volumes sharing the boundary. In the Modelicabased approach presented here, the Navier-Stokes equations are used to capture conservation of mass, momentum, energy and other associated transport phenomena for Newtonian fluids. The general form of all these equations for a conserved scalar density $q$ in a coordinate system at rest (not moving with the fluid) is given by [Versteeg and Malalasekera(1995)]:

$$
\underbrace{\partial_{t}(\rho q)}_{\begin{array}{c}
\text { local time } \\
\text { dependent } \\
\text { change }
\end{array}}+\underbrace{\operatorname{div}(\rho \vec{c} q)}_{\begin{array}{c}
\text { convective } \\
\text { term }
\end{array}}=\underbrace{\operatorname{div}(\Gamma \operatorname{grad} q)}_{\begin{array}{c}
\text { diffusive } / \\
\text { conductive } \\
\text { term }
\end{array}}+\underbrace{S_{q}}_{\begin{array}{c}
\text { source } \\
\text { term }
\end{array}}
$$

where " $\partial_{t}$ " is the partial derivative with respect to time, "div" represents the local divergence of a vector field and "grad" the local gradient vector of a scalar field. Moreover $\rho$ denotes the mass density, $\Gamma$ is a diffusion 
coefficient also known as conductivity in heat transport problems. The term $\vec{c}=(u, v, w)$ represents the flow velocity vector, which is the time derivative of the position vector $\vec{r}=(x, y, z)$ in a given coordinate system with coordinates $(x, y, z)$.

Each time derivative of each balance equation is a potential state in the jacobian matrix of the system model. Due to this fact the numerical effort for solving increases exponentially with the number air volumes in the grid model. Typically, up to 2.000 volumes can be handled with state-of-the-art work stations. Thus, the fluid dynamics library offers a coarse grid simulation. For a detailed view on the flow field a standard CFD simulation has to be carried out.

The spatial discretization (i.e., grid generation) of the air-conditioned space into cubic volumes is assisted through the free edition of the XRG Score Application (Microsoft Excel Addin), which creates the required geometry record (incl. view factors) for simulation. This Score edition is always shipped together with the FluidDynamics library. Moreover, it provides the post-processing of temperatures, and velocities in $2 \mathrm{~d}$ images (grid plot).

\section{Application to Fire Dynamics and Smoke Removal Simulation}

One possible application of the FluidDynamics Library is the usage for a fire dynamics and smoke removal simulation. In the following an exemplary use-case of a fire incident in an open-plane office is presented.

\subsection{Description of the Use case scenario}

The use-case scenario deals with a fire incident represented by an inflammation of a large-scale printer within a typical open-plan office with a floor space of $1000 \mathrm{~m}^{2}$ and workplaces for 64 people. Additionally, a kitchen counter is placed within the office. An overview of the office geometry is given in Figure 3. The installed fire-fighting system consists of automatic smoke detection devices as well as a mechanical smoke exhaust ventilation system.

The heat and smoke release can be described with a quadratic increasing curve until the maximum heat release rate is reached [VDI(2009)]. After that the heat and smoke release rate stays at a constant level. An appropriate value for the maximum heat release rate of a large-scale printer is $600 \mathrm{~kW}$. The smoke extraction system is sized with a volume flow rate of $60000 \mathrm{~m}^{3} / \mathrm{h}$ and is activated simultaneously with smoke detection.

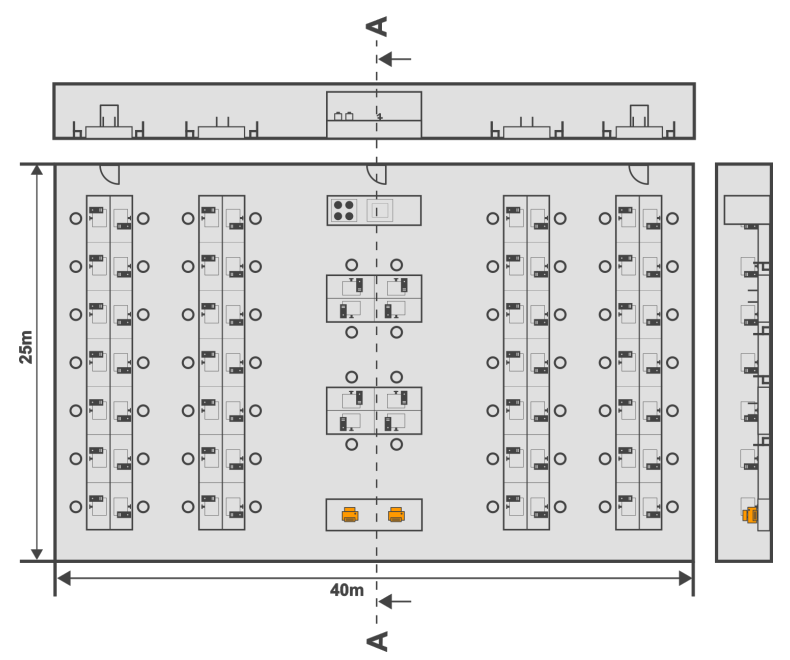

Figure 3. Overview about the office geometry

This can be assumed to be no later than 120 s after ignition according to [VDI(2009)]. Figure 4 shows the transient development of the heat release rate together with the smoke release flux and the exhaust air volume flow rate.

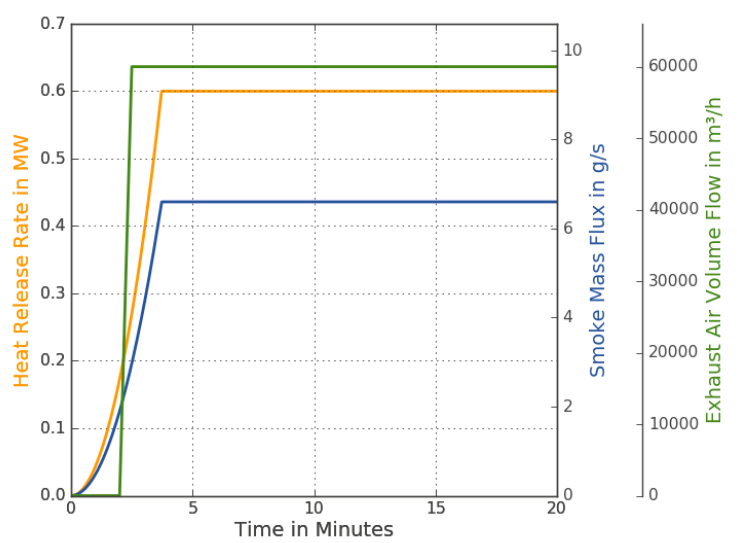

Figure 4. Course of heat and smoke release rate

The fire scenario shall be modeled and simulated both in CFD with ANSYS Fluent with a high locale resolution and with the Coarse Grid model of the FluidDynamics Library.

ANSYS Fluent is one of the most popular tools of the Computational Fluid Dynamics. The 3D-model of the office which is built in the ANSYS DesignModeler is displayed in Figure 5. The red volume is the source of fire. After activation of the smoke exhaust ventilation system the smoke is extracted via the 6 extraction points colored in orange. The green marked areas represent opening surfaces for fresh air entering the room in the case of fire. The air volume is meshed with the ANSYS Meshing Tool. The mesh consists of 4047834 cells in total. 


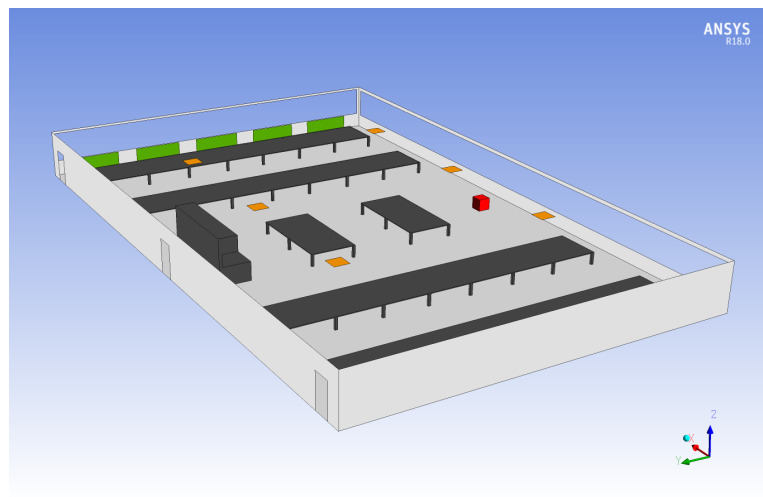

Figure 5. 3-D model of the office for CFD-Simulation with ANSYS Fluent

The Dymola/Modelica model of the same scenario using the FluidDynamics Library is displayed in Figure 6. It consists of the grid model of the room itself and further sub-components representing the main boundary conditions. A fire model computes the transient heat and smoke release within the grid. The air inlet model and 6 extraction models supply information about the ambient conditions at the opening surfaces and the volume flow rate at the extraction points. The coarse grid has a resolution of 13x8x9 hexagonal cells, so that in total 936 cells are used.

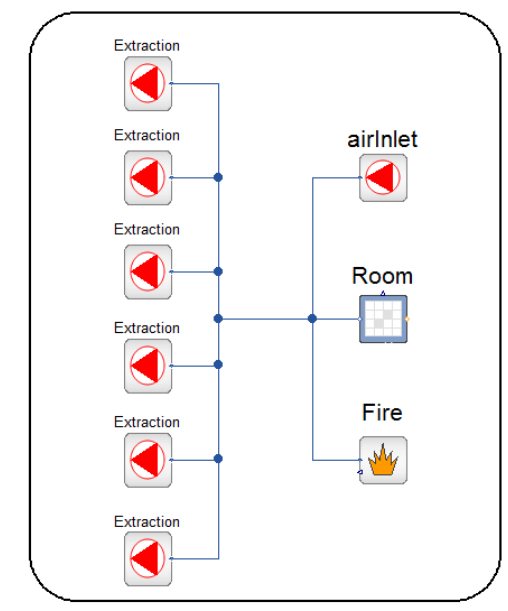

Figure 6. System model of the office for coarse grid simulation with the FluidDynamics Library

For the CFD-simulation with ANSYS Fluent a PC system with 8 cores (Intel(R) Core(TM) i7-6950X CPU @3,00 GHz) and 128GB RAM is used in parallel computation mode. The coarse grid simulation with the FluidDynamics Library in Dymola is proceeded with a PC with a Intel(R) Core(TM) i5-2500 CPU @ 3,30GHz processor and $16 \mathrm{~GB}$ RAM in single-core computation mode.

\subsection{Comparison of Simulation results}

In the following, the results of both the CFD-simulation in ANSYS Fluent and the Coarse-Grid simulation with the FluidDynamics Library are presented for the described fire incident within the office. Therefore the optical densities and air temperatures are visualized for both cases in cut A-A from Figure 3 for the exemplary moment of $600 \mathrm{~s}$ after the ignition of the fire. Moreover, the computation time for both cases is analyzed considering the used resources.

Figure 7 shows the local optical densities after 600s for cut A-A computed with ANSYS Fluent. One can see that the smoke has spread along the ceiling into the room. Moreover, clear layers of smoke and air can be noticed. The lowest point of the smoke layer is approximately $2,20 \mathrm{~m}$ above the floor. At the extraction points holes within the smoke layer can be found due to the so called plug-holing effect. Plug-holing describes the effect when air from beyond the smoke layer is sucked to the extraction points due to high vertical velocities at those positions which leads to the mentioned holes within the smoke layer.

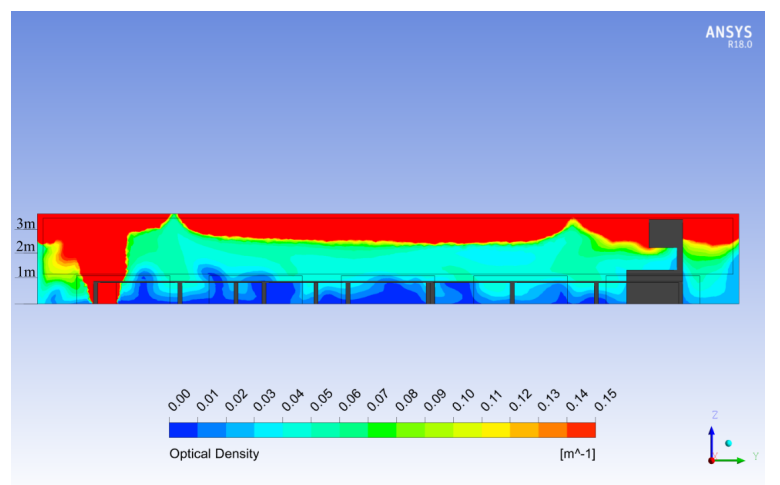

Figure 7. Local optical densities in cut A-A (see Figure 3) after 600s (ANSYS Fluent)

The local optical densities in cut A-A at 600s after ignition are displayed in Figure 8 for the results computed with the FluidDynamics Library in Dymola. It can also be seen that the smoke has spread along the ceiling into the room and a distinct smoke layer is existent. Compared to the result which is computed with ANSYS Fluent the local plug-holing effects are not visible. Nevertheless, a very similar thickness of the smoke layer can be stated. The lowest point of the smoke layer in the result created with the FluidDynamics Library is approximately $2,30 \mathrm{~m}$ above the floor.

In addition to the local optical densities the computed air temperatures shall be compared for both approaches. Figure 9 shows the local temperature after 600s for cut A-A computed with ANSYS Fluent. It is obvious 


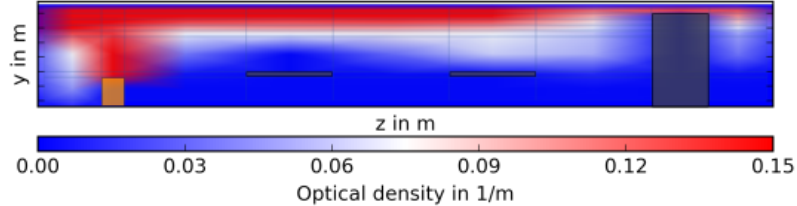

Figure 8. Local optical densities in cut A-A (see Figure 3) after 600s (FluidDynamics Library)

that the highest air temperatures can be found in the surrounding of the fire. The further away from the fire the lower are the temperatures in the smoke layer. The previously described plug-holing effect at the extraction points can also be seen in this temperature plot. In the areas in which workers are potentially present, temperatures only slightly above the ambient and starting temperature can be found.

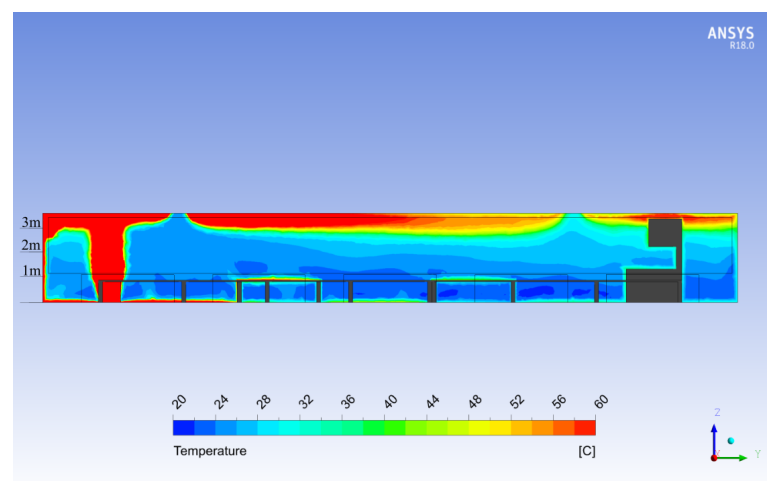

Figure 9. Local temperatures in cut A-A (see Figure 3) after 600s (ANSYS Fluent)

The temperature plot in Figure 10 shows the local temperatures in cut A-A for 600s after ignition, which are computed with the coarse grid model of the FluidDynamics Library. Generally, a very similar situation compared to the result of the CFD simulation with ANSYS Fluent can be seen. The highest temperatures in the smoke layer can also be found in the direct surrounding of the fire source. With increasing distance from the fire source the smoke layer temperatures decrease. In the lower zones in which people can be potentially present, the temperatures hardly exceed the starting room temperatures of $20^{\circ} \mathrm{C}$.

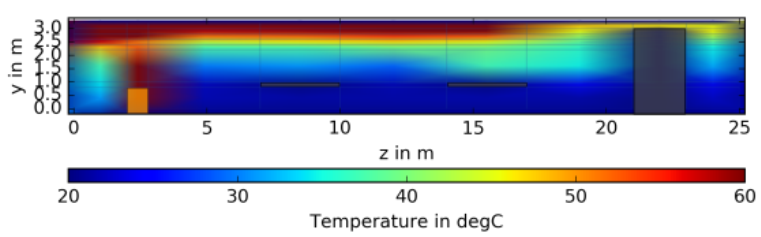

Figure 10. Local temperatures in cut A-A (see Figure 3) after 600s (FluidDynamics Library)
Considering the generally well matching results of both simulations, the computational effort is of special interest. As described above the CFD simulation with ANSYS Fluent is conducted with a multi-core PC in parallel computation mode while for the coarse grid simulation in Dymola a PC is used in single-core mode. While the CFD simulation can be started directly after initialization, the coarse grid model needs to be initialized dynamically fading in the physical effects step by step. This initialization process needs most of the computation time but only has to be done once, so that a simulation can be repeated with a modified set of input parameters with significantly less effort.

Figure 11 shows the time needed for computation for the single simulations. Comparing just the pure effort for one single simulation the coarse grid simulation with the FluidDynamics Library in Dymola took $21.4 \mathrm{~h}$ while $14.75 \mathrm{~h}$ were needed for the initialization process and $6.65 \mathrm{~h}$ for the simulation itself. The CFD simulation with ANSYS Fluent took 29.63h in total. Considering the differences in the hardware of 8 cores computing in parallel in the CFD simulation and one core being used in the coarse grid simulation it can be stated that the FluidDynamics approach needs significantly less resources for computation. This makes the usage of the coarse grid model of the FluidDynamics Library interesting for optimization processes, e.g. for finding a volume flow rate which is as small as possible to fulfill certain safety requirements.

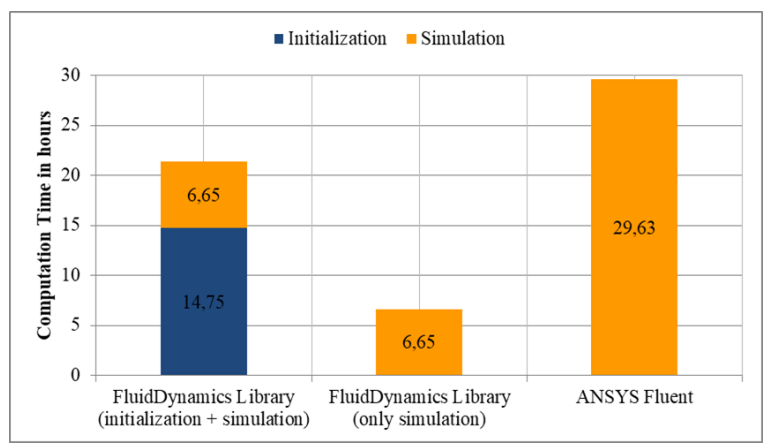

Figure 11. Comparison of computational effort

\section{Conclusions}

The comparison of the results of the simulated use case shows that the coarse grid approach of the FluidDynamics Library in Dymola delivers very similar results as the detailed CFD approach in ANSYS Fluent does. Even though local effects like plug-holing at extraction points are not captured in the coarse grid results the overall distribution of optical densities and air temperatures correspond very well to the ones computed with ANSYS Fluent. 
The significantly less computational effort of the coarse grid approach in the FluidDynamics Library makes it possible to conduct several simulations with the aim of optimization of e.g. necessary volume flow rates even with medium tier hardware. With this advantage the FluidDynamic Library can be used to plan, size and optimize smoke extraction systems in less time and with significantly lower costs than with detailed CFD tools and several iteration loops. In the process of approving a building permission this can cause a noticeable speed-up and also a significant reduction of costs due to the fact that the amount of necessary expensive CFD simulations can be limited to one single loop in the best case.

\section{References}

[VDI(2009)] Engineering methods for the dimensioning of systems for the removal of smoke from buildings. Verein Deutscher Ingenieure, 2009.

[MPC(2018)] Mpcci - multiphysics interface, 2018. URL https://www.mpcci.de/.

[TIS(2018)] Tisc suite, $2018 . \quad$ URL https: //www.tlk-thermo.com/index.php/en/ software-products/tisc-suite.

[Michaelsen(2015)] et al. Michaelsen. Dynamic simulation of an aircraft compartment coupled to a ventilation system. AST 2015, 2015.

[Versteeg and Malalasekera(1995)] H. Versteeg and W. Malalasekera. An introduction to computational fluid dynamics, The finite volume method. Longman Scientific \& Technical, 1995. 